\title{
Postoperative arterial blood lactate level as a mortality marker in patients with colorectal perforation
}

\author{
Jiro Shimazaki • Gyo Motohashi • Kiyotaka Nishida • \\ Hideyuki Ubukata • Takafumi Tabuchi \\ Accepted: 21 June 2013 / Published online: 12 July 2013 \\ (C) The Author(s) 2013. This article is published with open access at Springerlink.com
}

\begin{abstract}
Purpose This study aims to assess the clinical relevance of postoperative arterial blood lactate (LAC) level as a prognostic factor in patients with colorectal perforation.

Methods Forty-two patients (22 males, 20 females; mean age, 70.8 years) underwent emergency surgery for colorectal perforation. The patients were divided into mortality and survivor groups. As a prognostic scoring system, Acute Physiological and Chronic Health Evaluation II (APACHEII), Sequential Organ Failure Assessment (SOFA), and Systemic Inflammatory Response Syndrome criteria were calculated. These scores, postoperative LAC level, and other data, including site and etiology of perforation, elapsed time from onset to surgery (eTIME), preoperative white blood cell (WBC) and platelet counts, preoperative C-reactive protein (CRP), and preoperative arterial blood base excess were assessed between the groups.

Results The total mortality rate was $33.3 \%$. On univariate analysis, the APACHE-II and SOFA scores were significantly higher, and eTIME was significantly longer in the mortality group than in the survivor group. The postoperative LAC level was significantly higher in the mortality group $(43.1 \pm 14.1 \mathrm{mg} / \mathrm{dl})$ than in the survivor group $(23.8 \pm 12.7 \mathrm{mg} / \mathrm{dl} ; p<0.001)$, and the preoperative WBC was significantly lower in the mortality group than in the survivor group. Multivariate logistic regression analysis using the mortality risk factors determined by univariate analysis (eTIME, APACHE-II score, SOFA score, preoperative WBC count, and postoperative LAC) demonstrated that postoperative LAC level was an independent risk factor for mortality.
\end{abstract}

\footnotetext{
J. Shimazaki $(\bowtie) \cdot$ G. Motohashi $\cdot$ K. Nishida $\cdot$ H. Ubukata $\cdot$

T. Tabuchi

Department of Surgery, Ibaraki Medical Center, Tokyo Medical

University, 3-20-1, Chuo Ami-Machi, Inasiki-Gun, Ibaraki

300-0395, Japan

e-mail: shima-j@tokyo-med.ac.jp
}

Conclusions High postoperative LAC level was a useful factor for predicting high mortality rate in patients with colorectal perforation.

Keywords Colorectal perforation · Arterial blood lactate · Prognosis · Sepsis

\section{Introduction}

Colorectal perforation is a major life-threatening condition with high morbidity and mortality. This condition is responsible for generalized fecal peritonitis that can lead to septic shock, disseminated intravascular coagulation, and multiple organ failure. Clinically, evaluating the severity in patients with colorectal perforation and determining prognostic indicators is desirable to reduce the high mortality rate. There are many predictive scoring systems that show severity of serious illnesses, such as the Acute Physiological and Chronic Health Evaluation II (APACHE-II) [1], Sequential Organ Failure Assessment (SOFA) [2], and Physiological and Operative Severity Score for the enUmeration of Mortality and Morbidity [3]. Some studies have demonstrated that these scoring systems indicate the prognosis of patients with colorectal perforation, although there is no consensus. Furthermore, these scoring systems do not consider the arterial blood lactate (LAC) level, which predicts the poor prognosis of critically ill patients [4]. The blood LAC level increases in response to tissue hypoxia, and this level is currently used to monitor shock management [5-7]. Therefore, we reviewed our clinical experience with surgical and intensive treatment of colorectal perforation and retrospectively assessed the clinical relevance of postoperative arterial blood LAC level as a prognostic factor in patients with colorectal perforation. 


\section{Materials and methods}

Between 1998 and 2011, a 13-year period, 42 patients (22 males, 20 females; mean age, 70.8 years) underwent surgery for colorectal perforation at the Department of Surgery, Ibaraki Medical Center, Tokyo Medical University. The patients were divided into mortality and survivor groups. Patient medical records were reviewed, and clinical data, such as sex, age, site and etiology of perforation, elapsed time from onset to surgery (eTIME), preoperative white blood cell (WBC) and platelet counts, preoperative Creactive protein (CRP), preoperative arterial blood base excess (BE), and postoperative arterial blood LAC level, were collected. The postoperative LAC level was measured at the end of the operation; the APACHE-II score, SOFA score, and Systemic Inflammatory Response Syndrome (SIRS) criteria [8] were calculated postoperatively and compared between the mortality and survivor groups.

When appropriate, the data were presented as mean \pm SD. Stat Mate IV (ATMS Co. Ltd., Japan) was used to perform statistical analysis. The Mann Whitney $U$ test and the Pearson product-moment correlation coefficient were used to evaluate the correlations between variables in univariate analysis, and a logistic regression model was used to perform multivariate analysis. $p$ values $<0.05$ were considered statistically significant in all tests.

\section{Results}

The average age of the patients was $70.8 \pm 12.2$ years (range, $39-90$ years). The perforation sites were the rectum $(n=5)$, sigmoid colon $(n=21)$, transverse colon $(n=7)$, ascending colon $(n=3)$, cecum $(n=4)$, and appendix $(n=2)$. The operative procedures were Hartmann's operation $(n=22)$, colostomy $(n=9)$, right hemicolectomy $(n=3)$, ileocecal resection $(n=2)$, transverse colectomy $(n=2)$, direct closure $(n=2)$, and appendectomy $(n=2)$.

The total postoperative mortality rate was $33.3 \%$ (14 patients). Table 1 summarizes the preoperative clinical data and the APACHE-II score, SOFA score, and SIRS criteria for the mortality and survivor groups. The eTIME was significantly longer in the mortality group $(49.9 \pm 63.9 \mathrm{~h})$ than in the survivor group $(21.9 \pm 23.4 \mathrm{~h} ; p<0.05)$, and the mortality rate in the patients with eTIME $>12 \mathrm{~h}$ was $71.4 \%$ (10 patients). The APACHE-II and SOFA scores were significantly higher in the mortality group than in the survivor group $(21.2 \pm 7.69$ vs. $13.6 \pm 5.16, p<0.005 ; 9.1 \pm 2.8$ vs. $5.6 \pm 3.0, p<0.002$, respectively). Mortality was higher for the left-side colon perforation, including the descending colon to rectum, than for the right-side colon site, including the appendix to transverse colon ( 38.5 vs. $25.0 \%$, respectively), but the difference was not statistically significant. There were no statistically
Table 1 Clinical data between mortality and survivor groups

\begin{tabular}{llll}
\hline & Mortality $(n=14)$ & Survivors $(n=28)$ & $p$ value $^{\mathrm{a}}$ \\
\hline Age & $74.6 \pm 9.4$ & $68.7 \pm 13.2$ & n.s. \\
Male gender & 7 & 15 & n.s. \\
Perforation site & & & n.s. \\
$\quad$ Right side & $4(25.0 \%)$ & $12(75.0 \%)$ & \\
$\quad$ Left side & $10(38.5 \%)$ & $16(61.5 \%)$ & \\
Etiology & & & n.s. \\
$\quad$ Constipation & 9 & 10 & \\
$\quad$ Diverticulum & 1 & 7 & \\
$\quad$ Neoplasm & 3 & 6 & \\
Iatrogenesis & 0 & 2 & \\
Ischemia & 1 & 1 & $<0.05$ \\
Appendicitis & 0 & 2 & \\
eTIME (h) & $49.9 \pm 63.9$ & $21.9 \pm 23.4$ & \\
$\quad<12$ & $4(14.3 \%)$ & $24(85.7 \%)$ & \\
$\quad \geq 12$ & $10(71.4 \%)$ & $4(28.6 \%)$ & \\
APACHE-II score & $21.2 \pm 7.69$ & $13.6 \pm 5.16$ & $<0.005$ \\
SOFA score & $9.1 \pm 2.8$ & $5.6 \pm 3.0$ & \\
SIRS criteria & $1.86 \pm 1.03$ & $1.68 \pm 1.12$ & \\
\hline
\end{tabular}

eTIME elapsed time from onset to surgery, APACHE-II Acute Physiological and Chronic Health Evaluation II, SOFA Sequential Organ Failure Assessment, SIRS systemic inflammatory response syndrome, n.s., not significant

${ }^{\text {a }}$ The Mann-Whitney $U$ test was applied

significant differences in the mortality rate between age, gender, site, and etiology of perforation (Table 1).

Table 2 presents a comparison of the preoperative laboratory data, including WBC and platelet counts, CRP level, BE level, and postoperative LAC level between the mortality and survivor groups. The mortality rate was higher in the patients with preoperative WBC count $<4,000 / \mu$ l than in those with preoperative WBC count $\geq 4,000 / \mu$ l (52.9 vs. $20.0 \%$, respectively; $p<0.05$ ). The postoperative LAC level was significantly higher in the mortality group than in the survivor group $(43.1 \pm 14.1$ vs. $23.8 \pm 12.7 \mathrm{mg} / \mathrm{dl}$, respectively; $p<0.001)$. There were no statistically significant differences in the mortality rate between the preoperative platelet count, CRP levels, and BE levels (Table 2).

Multivariate logistic regression analysis using the mortality risk factors determined by univariate analysis (eTIME, APACHE-II score, SOFA score, preoperative WBC count, and postoperative LAC level) showed that the postoperative LAC level was an independent risk factor for mortality in the patients with colorectal perforation (odds ratio, 1.104; $95 \%$ confidence interval, 1.006-1.214; $p=0.037$ ) (Table 3). Figure 1 shows statistical regression line between the postoperative LAC levels and total mortality rate, and there was a statistically significant correlation between them $(p<0.001)$ 
Table 2 Preoperative and postoperative laboratory data between mortality and survivor groups

$V B C$ white blood cell, $C R P C$ reactive protein, $B E$ base excess, $L A C$ lactate, n.s. not significant

${ }^{\text {a }}$ The Mann-Whitney $U$ test was applied

\begin{tabular}{llll}
\hline & Mortality $(n=14)$ & Survivors $(n=28)$ & $p$ value $^{\mathrm{a}}$ \\
\hline $\begin{array}{l}\text { Preoperative WBC count }(\text { per } \mu \mathrm{l}) \\
\quad<4,000\end{array}$ & $52.9 \%(n=9)$ & $47.1 \%(n=8)$ & $<0.05$ \\
$\quad \geq 4,000$ & $20.0 \%(n=5)$ & $80.0 \%(n=20)$ & \\
Preoperative platelet count $\left(\times 10^{4} / \mu \mathrm{l}\right)$ & $28.4 \pm 7.4$ & $29.3 \pm 8.5$ & \\
Preoperative CRP $(\mathrm{mg} / \mathrm{dl})$ & $9.23 \pm 3.73$ & $8.26 \pm 4.35$ & n.s. \\
Preoperative BE & $-4.57 \pm 5.94$ & $-0.59 \pm 4.19$ & n.s. \\
Postoperative LAC $(\mathrm{mg} / \mathrm{dl})$ & $43.1 \pm 14.1$ & $23.8 \pm 12.7$ & $<0.001$ \\
\hline
\end{tabular}

(Fig. 1). The regression line could be used to predict the total mortality rate from the postoperative LAC level.

\section{Discussion}

The frequency of colorectal perforation is lower than that of upper gastrointestinal tract perforation, but its mortality rate is as high as 15-30\% [9-12]. Colorectal perforation rapidly leads to diffuse peritonitis and septic shock due to the presence of large amounts of gram-negative bacilli in intestinal fluid and causes multiple organ failure and acute circulatory failure due to the release of chemical mediators such as endotoxins and inflammatory cytokines interleukin (IL)-1 and IL-6. Therefore, to improve the prognosis of colorectal perforation, it is important to accurately determine the status of peritonitis, the severity of the pathological condition, and any prognostic factors. Several reports have investigated on the prognostic factors for colorectal perforation. Our study suggests that a longer elapsed time from onset to surgery increases the mortality rate and strongly emphasizes the importance of early diagnosis and treatment for improving the survival rate. The mortality rate was high in patients with preoperative WBC counts of $<4,000 / \mu l$. In general, decreased WBC count is observed in cases where long durations of time have elapsed following the onset of peritonitis and when basic immune function is compromised because of aging $[9,13]$. These conditions involve utilization of a high number of leukocytes as a biological reaction to severe inflammation. No statistically significant differences were found between different perforation sites, although the mortality rate tended to be higher in perforations located on the left side of the colon than those on the right side of the colon. This was considered to be due to localized differences in the bacterial colonies and amount of bacteria in the colon.

APACHE-II and SOFA scores have been proposed as prognostic predictors in severe illnesses. APACHE-II scores are used as an index for patient severity assessments when they are admitted to the intensive care unit. SOFA scores quantify the degree of organ failure and determine the mortality rate on the basis of six items, including the respiratory, coagulation, cardiovascular, and central nervous systems as well as renal and hepatic functions. Whether these scores are prognostic predictors for colorectal perforation is debatable. Horiuchi et al. [14] reported on the usefulness of APACHE-II scores as a prognostic predictor in that significantly high scores were related to the mortality rate of patients with colorectal perforation. In addition, Komatsu et al. [9] reported that the mortality rate was significantly higher for patients with APACHE-II scores of $\geq 19$ or SOFA scores of $\geq 8$. In this study, we focused on the APACHE-II and SOFA scoring systems and SIRS criteria. When mortality and survivor groups of patients with colorectal perforation were compared, a relationship between APACHE-II and SOFA scores and mortality rate was observed using univariate analysis. The SIRS criteria assess the four items of body temperature, heart rate, respiratory status, and leukocyte count. These items indicate the extent of the body's immune response to infection and are mainly used for assessing septic shock. Colorectal perforation progresses to sepsis early because of the pathological condition; therefore, we suspected that the SIRS criteria and mortality rate may be associated, although this association could not be established. The SIRS criteria are used to assess septic shock, but do not reflect

Table 3 Multivariate analysis of the independent risk factors for mortality

\begin{tabular}{llll}
\hline Variables & Odds ratio & $95 \%$ Confidence interval & $p$ value $^{\mathrm{a}}$ \\
\hline eTIME & 1.028 & $0.988-1.069$ & $\mathrm{~ns}(0.169)$ \\
APACHE-II score & 1.046 & $0.863-1.267$ & $\mathrm{~ns}(0.645)$ \\
SOFA score & 1.122 & $0.767-1.642$ & $\mathrm{~ns}(0.551)$ \\
Preoperative WBC & 0.999 & $0.999-1.000$ & $\mathrm{~ns}(0.754)$ \\
Postoperative LAC & 1.104 & $1.006-1.214$ & 0.037 \\
\hline
\end{tabular}

eTIME elapsed time from onset to surgery, APACHE-II Acute Physiological and Chronic Health Evaluation II, SOFA Sequential Organ Failure Assessment, $W B C$ white blood cell, $L A C$ lactate, $n s$ not significant

${ }^{\mathrm{a}}$ Multivariate logistic regression was applied 
Fig. 1 Statistical regression line between the postoperative LAC levels and total mortality rate

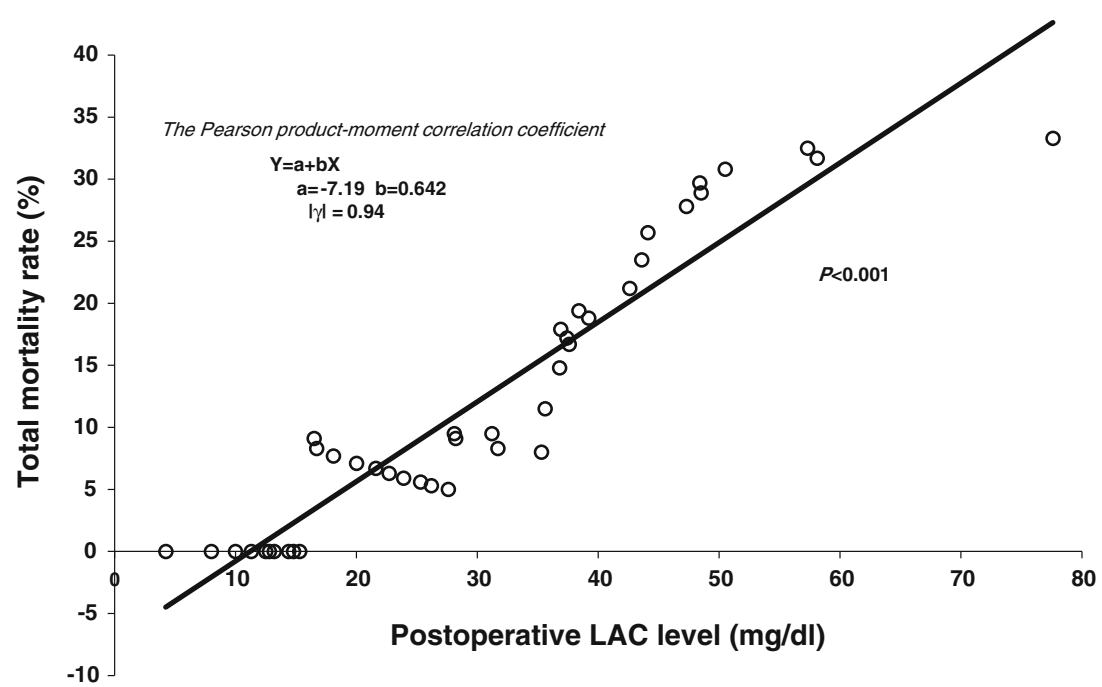

pathological changes such as organ failure and acute circulatory failure that occur later. This probably explains why we could not establish an association between the SIRS criteria and mortality rate.

In this study, we also focused on arterial blood LAC levels and examined its usefulness as a prognostic factor for patients with colorectal perforation. LAC levels are not included in the current severity assessment scoring systems, including APACHE-II and SOFA, and there have been no reports on possible relationships between the prognosis of patients with colorectal perforation and their LAC levels. LAC levels reflect organ failure due to lack of tissue oxygenation [15]. In the clinical setting, patients with severe illnesses have high LAC levels. LAC levels are used for the prognostic assessment of septic shock and acute respiratory distress syndrome and are useful for determining the therapeutic outcomes and changes in pathological conditions [6, $16,17]$. In recent years, there have been reports on the use of LAC level as a prognostic factor for high technical surgery, particularly cardiovascular surgery [18-20]. Based on these reports, we speculated that LAC levels could be used as a prognostic factor for patients with colorectal perforation that rapidly causes sepsis. In this study, we found that the mortality rate was high among patients with high postoperative LAC level on univariate analysis. Furthermore, in a multivariate analysis using the mortality risk factors determined by univariate analysis, LAC level was an independent risk factor of mortality. Although the odds ratio for postoperative LAC levels was low, these results suggested that predicting the postoperative mortality rate based on the LAC levels immediately after surgery was possible using the regression line obtained. LAC level was an independent risk factor in our multivariate analysis as opposed to the APACHE-II and SOFA scores because the APACHE-II scores incorporate an assessment item for acidosis, but not for LAC levels. Moreover, the SOFA scores incorporate an assessment item for organ failure, but do not include one for acidosis. Gunnerson et al. [21] reported that acidosis caused by elevated LAC levels has been associated with high mortality than acidosis due to other underlying causes in severe illness. Therefore, in severe illness such as colorectal perforation, assessing LAC levels is important. LAC level is an easily measurable marker of decreased peripheral perfusion. Whether preoperative LAC levels or postoperative LAC levels reflect the prognosis of patients with colorectal perforation remains debatable. In this study, we examined LAC levels immediately after surgery, taking into account surgical stress, and LAC levels were not measured during the preoperative examination of colorectal perforation during this study period. Taking the results of our study into account, we have begun measuring preoperative LAC levels and are investigating their usefulness as a prognostic factor.

\section{Conclusion}

To reduce the high mortality of colorectal perforation, it is essential to make an early diagnosis and to have surgery as soon as possible. The postoperative arterial blood lactate level is a useful factor for predicting high mortality rate in patients with colorectal perforation.

Acknowledgments The authors would like to thank Enago (www. enago.jp) for the English language review.

Conflict of interest The authors declared no conflicts of interest and financial disclosure with respect to the authorship and/or publication of this article. 
Open Access This article is distributed under the terms of the Creative Commons Attribution License which permits any use, distribution, and reproduction in any medium, provided the original author(s) and the source are credited.

\section{References}

1. Knaus WA, Draper EA, Wagner DP, Zimmerman JE (1985) APACHE II: a severity of disease classification system. Crit Care Med 13(10):818-829

2. Vincent JL, Moreno R, Takala J, Willatts S, De Mendonca A, Bruining H, Reinhart CK, Suter PM, Thijs LG (1996) The SOFA (Sepsis-related Organ Failure Assessment) score to describe organ dysfunction / failure. On behalf of the Working Group on SepsisRelated Problem of the European Society of Intensive Care Medicine. Intensive Care Med 22(7):707-710

3. Copeland GP, Jones D, Walters M (1991) POSSUM: a scoring system for surgical audit. Br J Surg 78(3):355-360

4. Khosravani H, Shahpori R, Stelfox HT, Kirkpatrick AW, Laupland KB (2009) Occurrence and adverse effect on outcome of hyperlactatemia in the critically ill. Crit Care 13(3):R90

5. Kurimoto Y, Kawaharada N, Ito T, Morikawa M, Higami T, Asai Y (2008) An experimental evaluation of the lactate concentration following mesenteric ischemia. Surg Today 38(10):926-930

6. Yang CS, Qiu HB, Huang YZ, Xie JF, Mo M, Liu SQ, Yang Y (2009) Prospective research on the prognosis of septic shock based on the change of lactate concentration in arterial blood. Zhonghua Wai Ke Za Zhi 47(9):685-688

7. Pattharanitima $\mathrm{P}$, Tongyoo S, Ratanarat R, Wilachone $\mathrm{W}$, Poompichet A, Permpikul C (2011) Correlation of arterial, central venous and capillary lactate levels in septic shock patients. J Med Assoc Thai 94(Suppl 1):S175-180

8. Bone RC, Balk RA, Cerra FB, Dellinger RP, Fein AM, Knaus WA, Schein RM, Sibbald WJ (1992) Definitions for sepsis and organ failure and guidelines for the use of innovative therapies in sepsis. The ACCP/ SCCM Consensus Conference Committee. American College of Chest Physicians/Society of Critical Care Medicine. Chest 101(6):1644-1655

9. Komatsu S, Shimomatsuya T, Nakajima M, Amaya H, Kobuchi T, Shiraishi S, Konishi S, Ono S, Maruhashi K (2005) Prognostic factors and scoring system for survival in colonic perforation. Hepatogastroenterology 52(63):761-764

10. Shinkawa H, Yasuhara H, Naka S, Yanagie H, Nojiri T, Furuya Y, Ariki K, Niwa H (2003) Factors affecting the early mortality of patients with nontraumatic colorectal perforation. Surg Today 33(1):13-17

11. Bielecki K, Kaminski P, Klukowaski M (2002) Large bowel perforation: morbidity and mortality. Tech Coloproctol 6(3):177-182

12. Kriwanek S, Armbruster C, Beckerhinn P, Dittrich K (1994) Prognostic factors for survival in colonic perforation. Int $\mathrm{J}$ Colorectal Dis 9(3):158-162

13. Aminzadeh Z, Parsa E (2011) Relationship between age and peripheral white blood cell count in patients with sepsis. Int $\mathrm{J}$ Prev Med 2(4):238-242

14. Horiuchi A, Watanabe Y, Doi T, Sato K, Yukumi S, Yoshida M, Yamamoto Y, Sugishita H, Kawachi K (2007) Evaluation of prognostic factors and scoring system in colonic perforation. World $\mathrm{J}$ Gastroenterology 13(23):3228-3231

15. Nielsen C, Mortensen FV, Erlandsen EJ, Lindholt JS (2012) L- and D-lactate as biomarkers of arterial-induced intestinal ischemia: an experimental study in pigs. Int J Surg 10(6):296-300

16. Wu WH, Niu YY, Zhang CR, Xiao LB, Ye HS, Pan DM, Zeng M (2012) Combined APACH- II score and arterial blood lactate clearance rate to predict the prognosis of ARDS patients. Asian Pac J Trop Med 5(8):656-660

17. Kjelland CB, Djogovic D (2010) The role of serum lactate in the acute care setting. J Intensive Care Med 25(2):286-300

18. Basaran M, Sever K, Kafali E, Ugurlucan M, Sayin OA, Tansel T, Alpagut U, Dayioglu E, Onursal E (2006) Serum lactate level has prognostic significance after pediatric cardiac surgery. J Cardiothorac Vasc Anesth 20(1):43-47

19. Rocha TS, Silveria AS, Botta AM, Ricachinevsky CP, Dalle Mulle L, Nogueira A (2010) Serum lactate as mortality and morbidity marker infants after Jatene's operation. Rev Bras Cir Cardiovasc 25(3):350-358

20. Hashimoto M, Ito T, Kurimoto Y, Harada R, Kawaharada N, Higami T (2012) Preoperative arterial blood lactate levels as a predictor of hospital mortality in patients with a ruptured abdominal aortic aneurysm. Surg Today. doi:10.1007/s00595-012-0439-7

21. Gunnerson KJ, Saul M, He S, Kellum JA (2006) Lactate versus non-lactate metabolic acidosis: a retrospective outcome evaluation of critically ill patients. Crit Care 10(1):R22 